\title{
Ultrawide band gap design of phononic crystals based on topological optimization
}

\author{
Kai Wang ${ }^{\mathrm{a}}$, Ying Liu ${ }^{\mathrm{b} *}$, Bin Wang \\ ${ }^{a}$ Department of Mechanics, School of Civil Engineering, Beijing Jiaotong University, Beijing 100044, \\ PR China \\ ${ }^{b}$ School of civil Engineering, Beijing Jiaotong University, Beijing 100044 PR China \\ Email: yliu5@bjtu.edu.cn; Tel: 86-10-51688763; Fax: 86-10-51682094 \\ ${ }^{\mathrm{c}}$ Department of Mechanics and Aerospace Engineering, Brunel University London, Kingston Lane, \\ Uxbridge UB8 3PH, United Kingdom
}

\begin{abstract}
In this paper, based on the combined finite element method and bidirectional evolutionary structural optimization algorithm, we perform the topological optimization of phononic crystals to obtain ultrawide band gap between two special adjacent bands for both the in-plane and out-of-plane wave modes. The influences of matrix/scatter materials, material volume fraction, and initial topological design on the unit cell optimization are discussed in detail. The results show that the strategy proposed in this paper is effective and efficient to obtain better optimization results under the similar optimization condition, solutions with ultrawide band gaps can be easily obtained within 30 iterations. Several new patterns for phononic band gap crystals with optimized band gaps are presented. This work provides useful guidance in topological optimization design of phononic
\end{abstract}


crystals.

Keywords: Ultrawide band gap; Bidirectional evolutionary structural optimization; Phononic crystals; Finite element method; Topology optimization;

\section{Introduction}

As one kind of functional periodic structures, phononic crystals (PnCs) were firstly proposed in 1993 [1]and had become a hot research topic due to their distinctive physical features for numerous practical applications. One of the most important and useful physical properties of PnCs is the existence of the absolute band gaps (ABGs)[2], within which the elastic waves propagation is prevented or significantly weakened. Due to this special physical characteristic, phononic crystals attract increasing attentions in multi-functional fields[3-7], particularly in acoustic fields where tuning of bandgap in PnCs becomes more and more essential.

For a given filling fraction, apart from the contrast of physical parameters between the scatter and the matrix, the topological structure of the unit cell is also a key issue to the formation of ABGs in PnCs[8-13]. Up to now, many efforts have been paid to design PnCs aiming to find optimized ABGs based on optimized topological structures. Liu et al.[14, 15] analyzed the influence of the lattice transformation and lattice structures on the ABGs in PnCs, and noticed a significant influence of cell topology on ABGs. Yang et al.[16] analyzed the spherical three- dimensional (3D) nano-scale silicon PnCs and the influence of the porosity is considered as well. Wang et al.[17-19] proposed a Sierpinski triangle fractal and grading strategies in the topological structure design and obtained some larger band gaps. Optimized unit cell with excellent bandgap performance arouses a great deal of interest on the design of PnCs [20-22].

Since the pioneering topology optimization work was developed by Kikuchi and Bendsoe [23], a lot of effective optimization algorithms were proposed, such as level set method (LSM)[24, 25]; solid isotropic material with 
penalization (SIMP)[26, 27]; evolutionary structural optimization (ESO)[28]; bi-directional evolutionary structural optimization (BESO)[29, 30]; and the moving morphable components method (MMC)[31, 32], which have been widely used to deal with topological problems including structural, optical or acoustic performances[33, 34]. Recently, ABG optimization in PnCs attracts great attention. Using the method of moving asymptotes, Sigmund and Jensen[35] studied the topology optimization work of PnCs. Diaz et al.[36] performed the maximal design of ABGs and they discussed the influence of the skew angle of the underlying grid structure on band gaps. Halkjer et al.[37] combined FEM with moving asymptotes method and they maximized the band gap in a Mindlin plate. Based on the genetic algorithm which was proposed by Holland[38], various work has been devoted to optimize topological structure of PnCs. Hussein et al.[39, 40] conducted a series of optimization studies for maximizing band gaps in onedimensional PnCs, PnC-based filter design and a vacuum/solid porous PnC, respectively. Based on a "coarse to fine" two-stage genetic algorithm, Dong et al.[41] conducted a PnC optimization with the unconstrained problem. Based on BESO, Huang and Li[42] performed the band optimization in PnCs using a modified SIMP model[43]. More effective and efficient algorithm is always the frontier in band optimization of PnCs.

Focusing on the robustness and computational efficiency, a combined BESO and finite element scheme is proposed to perform the topology optimization in 2D square-latticed solid PnCs for the maximum ABG between two specified adjacent bands. The BESO procedure is programmed as a part of the finite element method (FEM) software package and become very simple and easily implemented. The advantage of this new BESO/FEM combined strategy to other optimization algorithm is discussed in detail. At last, the conclusion is given.

\section{Waves in phononic crystals}

Figure 1 shows a typical representation of a 2D solid-solid phononic crystal and the corresponding first Brillouin zone. Circular scatters which are made by Material 2 (denoted by A) periodically located in the matrix which is made by Material 1 (denoted by B). A unit cell is marked out by the dashed lines with a lattice length a. The irreducible 
Brillouin zone of the lattice $\Gamma \mathrm{XM}$ is marked as the shaded area.
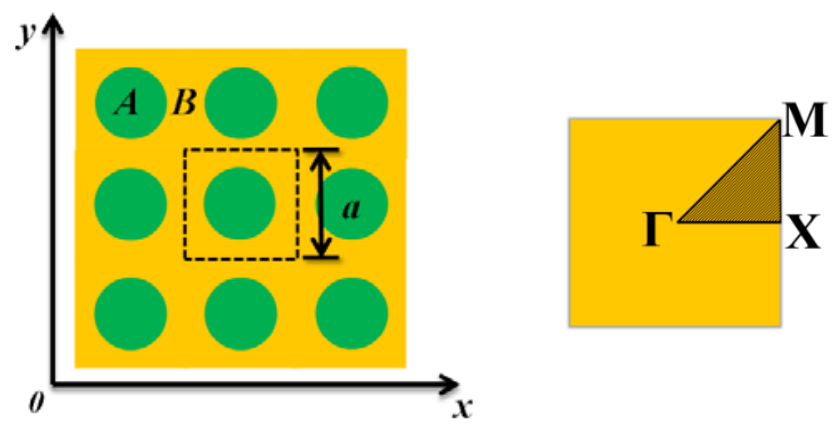

Fig. 1. Diagrammatic sketch of a representative 2D solid-solid phononic crystal and the first Brillouin zone of the 2D square lattice.

The $z$-coordinate is set parallel to the scatter. In this case, the elastic waves propagate in the xoy plane can be decoupled into the anti-plane shear mode and the mixed in-plane mode. Due to the $z$ coordinate and the xoy plane are perpendicular to each other, displacement vectors in the in-plane mode are independent of the $z$-coordinate, which are given as

$$
\begin{gathered}
-\rho(\mathbf{r}) \omega^{2} u_{e}=\nabla \cdot\left[\mu(\mathbf{r}) \nabla u_{e}\right]+\nabla \cdot\left[\mu(\mathbf{r}) \frac{\partial}{\partial x_{e}} \mathbf{u}\right]+\frac{\partial}{\partial x_{e}}[\lambda(\mathbf{r}) \nabla \cdot \mathbf{u}] \quad(e=x, y), \\
-\rho(\mathbf{r}) \omega^{2} u_{z}=\frac{\partial}{\partial x}\left[\mu(\mathbf{r})\left(\frac{\partial u_{z}}{\partial x}\right)\right]+\frac{\partial}{\partial y}\left[\mu(\mathbf{r})\left(\frac{\partial u_{z}}{\partial y}\right)\right] .
\end{gathered}
$$

Here, $\nabla=(\partial x, \partial y)$ and $\mathbf{u}=\left(u_{x}, u_{y}\right)$ is the transverse plane displacement vector. In a periodic structure, based on the Bloch theorem, the displacement filed can be expressed as follows [44].

$$
\mathbf{u}(\mathbf{r})=\mathrm{e}^{\mathrm{i}(\mathbf{k} \cdot \mathbf{r})} \mathbf{u}_{\mathbf{k}}
$$

where $\mathrm{i}$ is the imaginary unit, $\mathbf{u}_{\mathbf{k}}(\mathbf{r})$ is a periodical vector function with the same periodicity as the crystal lattice, and $\mathbf{k}=\left(k_{x}, k_{y}\right)$ is the Bloch wave vector, which is limited to the first Brillouin zone of the reciprocal lattice.

In the unit cell, the eigenvalue equations can be expressed as

$$
\left(\mathbf{K}-\omega^{2} \mathbf{M}\right) \mathbf{U}=0
$$

where $\mathbf{U}$ is the nodes displacement, $\mathbf{K}$ is the global stiffness matrix, $\mathbf{M}$ is the global mass matrix of the unit cell, 
Following Bloch conditions on the two opposite boundaries of a unit cell, the displacement field are given as:

$$
\mathbf{U}(\mathbf{r}+\mathbf{a})=\mathrm{e}^{i(\mathbf{k a})} \mathbf{U}(\mathbf{r}),
$$

where $\mathbf{r}$ is the position vector at the boundary nodes, $\mathbf{a}$ is the lattice basis vector associated with the PnCs.

By applying Eq. 5. into the complex boundary condition, we used COMSOL with MATLAB to solve the eigenvalues of Eq. (4). The model was built in FEM and then saved as a MATLAB-compatible '.m' file. Triangular Lagrange quadratic elements were utilized to mesh the unit cell. The direct SPOOLES (SParse Object Oriented Linear Equations Solver) was used under Eigen-frequency analysis. We programmed the '.m’ file to let the wave vector $\mathrm{k}$ sweep along the edges of the irreducible Brillouin zone. Then the dispersion relations of PnCs can be obtained. Based on the secondary development of the FEM program, we can obtain the width of the aimed ABG and then make the bandwidth optimization based on the BESO algorithm.

\section{BESO/FEM combined strategy}

The basic concept of BESO is that it can evolve structures towards an optimum by systematically adding efficient elements meanwhile removing inefficient ones. BESO has been successfully used in solving problems in many fields which demonstrated the reliability and efficiency of BESO in structural optimization. In the following section, we introduce the proposed BESO/FEM combined strategy with the aim to opening and maximizing the ABG between two specified bands for given matrix/scatter materials.

\subsection{Statement of objective and constraint functions}

As a kind of functionally periodic structures, the topology of 2D solid-solid phononic crystal can be expressed by one of the repeating unit cells. The optimization aim here is to obtain the optimized unit cell which has the maximum ABG between two given adjacent bands. The ABG bandwidth is transformed to the dimensionless form by its central frequency. Thus, this optimization problem can be mathematically described as an objective function 
as follows:

$$
\text { Maximize: } f\left(\sum\right)=2 * \frac{\min _{\mathbf{k}}: \omega_{\mathrm{n}+1}\left(\sum, \mathbf{k}\right)-\max _{\mathbf{k}}: \omega_{\mathrm{n}}\left(\sum, \mathbf{k}\right)}{\min _{\mathbf{k}}: \omega_{\mathrm{n}+1}\left(\sum, \mathbf{k}\right)+\max _{\mathbf{k}}: \omega_{\mathrm{n}}\left(\sum, \mathbf{k}\right)},
$$

where $f$ denotes the relative dimensionless ABG which is simplified by the central frequency between the given pair of adjacent bands, $\sum$ is the topological distribution of one repeating unit cell. $\omega_{\mathrm{n}}$ is the frequency of the $\mathrm{n}^{\text {th }}$ energy band. The orders of the aim bandgaps are extracted by $n$.

In this work, we only consider the repeating unit cell within a square lattice. The unit cell can be molded by $\mathrm{N} \times \mathrm{N}$ square pixels. We define the discrete design variable $x_{p}(p=1,2,3 \ldots)$ for each pixel to describe the topology structure of the unit cell. $x_{p}=0$ represents the $p^{\text {th }}$ pixel being made of Material 1 (matrix), and $x_{p}=1$ for Material 2 (scatter). It is known that due to the interpolation, the discrete design variable may appear intermediate values, which may result in 'grey' elements in the resulted structures[34]. In order to avoid this problem, in our algorithm, $x_{p}$ is set to be 1 or 0 so that the optimized solutions will convergent to absolute values, 1 or 0 , avoiding the 'grey' element as shown in our numerical examples.

For practical application of phononic crystals, proportioning of different materials in PnCs always needs to be considered. In this case, volume fraction of Material 2 was selected to be the constraint function.

$$
\text { Subject to } \quad \frac{\sum_{i=1}^{\mathrm{N}} v_{i} x_{i}}{\mathrm{~V}^{\mathrm{N}}}=\mathrm{V}_{f}^{*},\left(x_{i}=0 \text { or } 1\right)
$$

where $v_{p}$ denotes the volume of element $p, \mathrm{~N}$ is the total number of the square pixels in the unit cell. $\mathrm{V}_{f}^{*}$ is the prescribed volume fraction of Material 2.

\subsection{Volume evolution and sensitivity analysis}

In our procedure, the BESO algorithm starts from a unit cell made from Material 1. Based on the volume evolution, the volume fraction of Material 2 will increase from 0 to the prescribed volume $\mathrm{V}_{f}^{*}$ following:

$$
\mathrm{V}_{h+1}=\mathrm{V}_{h}(1+\mathrm{ER}) \quad \text { when } \mathrm{V}_{h+1} \leq \mathrm{V}_{f}^{*}(h=1,2,3 \ldots)
$$


where $h$ is the iteration number, ER denotes the evolutionary volume ratio and. $V_{h}$ is the current volume of Material 2 in the $h^{\text {th }}$ iteration. In our BESO procedure, the there is no Material 2 in the initial design which means $V_{1}=0$. In order to start the volume evolution and control the minimal evolution speed of the procedure, ER is set to 2 and the minimum variation of the structure is set to 2 square pixels. At the updated volume $V_{h+1}$, optimization iterations are made until both the objective function and volume constraint function are stably converged and satisfied.

In analyzing a design structure $\sum=\left\{x_{1}, x_{2}, \ldots, x_{p} \ldots, x_{n}\right\}^{\mathrm{T}}$ to have the function $f\left(\sum\right)$, where $\sum$ is the material distribution defined by discrete design variable $x_{p}$, if there is a disturbance $\Delta x_{p}$ on the $p^{\text {th }}$ design variable, a new design structure $\sum+\Delta \sum=\left\{x_{1}, x_{2}, \ldots, x_{p}+\Delta x_{p} \ldots, x_{n}\right\}^{\mathrm{T}}$ would be obtained. The sensitivity of the structure can be expressed as:

$$
\alpha_{p}^{e}=\frac{\partial f}{\partial \sum_{p}}=\frac{f\left(\sum+\Delta \sum_{p}\right)-f\left(\sum\right)}{\Delta \sum_{p}}
$$

In order to simplify the calculation of sensitivity numbers, consider the centrosymmetry of the unit cell, only a quarter of the unit cell is modeled. We applied one perturbation to the structure on each square pixel with $x_{p}=0$ (made of Material 1). Based on Eq. (9) and FE calculation, the initial sensitive number of all square pixels with $x_{p}$ $=0$ could be obtained .

In BESO method, material can be removed and added simultaneously. Material removal and addition scheme ranks all pixels together based on their sensitive numbers. $x_{i}$ of pixels with high sensitive numbers will be set to 1 to add Material 2; meanwhile $x_{i}$ of pixels with low sensitive numbers will be set to 0 to add Material 1 . Due to the symmetry, there may be some same highest sensitive numbers occurs at the same iteration, when it happens, the proposed BESO/FEM procedure will randomly select one of the largest ones to add material meanwhile break the symmetry. Eq. (9) gives sensitive numbers for square pixels with $x_{i}=0$ (made of Material 1). A filter scheme is further used here to extrapolate sensitivity numbers for pixels with $x_{i}=1$. 


\subsection{Filter scheme and BESO/FEM procedure}

As a smoothing strategy, Filter technique can not only calculate sensitivity numbers, but also suppress some numerical instability such as mesh-dependency or checkerboard [45-47]. Before applying the filter scheme[48], we calculate the nodal sensitivity numbers of each pixel with $x_{\mathrm{p}}=0$ as follows:

$$
\alpha_{p}^{\mathrm{n}}=\sum_{j=1}^{\mathrm{M}} \omega_{j} \alpha_{j}^{\mathrm{e}}
$$

where $\mathrm{M}$ is the total number of pixels which are connected to the $p^{\text {th }}$ pixel with $x_{p}=0 . \alpha_{j}^{\mathrm{e}}$ is the initial sensitive number obtained by Eq. (9). $\omega_{j}$ donates the weight factor of the $j^{\text {th }}$ pixel defined as

$$
\omega_{j}=\frac{1}{\mathrm{M}-1}\left(1-\frac{r_{p j}}{\sum_{j=1}^{\mathrm{M}} r_{p j}}\right),
$$

where $r_{p j}$ is the distance between the centers of the pixel $p$ and pixel $j$ (Fig.2).

Based on the nodal sensitivity numbers obtained from Eq. (10), the modified sensitivity number of the $p^{\text {th }}$ pixel is given as[48]:

$$
\alpha_{p}^{m}=\frac{\sum_{\mathrm{j}=1}^{\mathrm{N}} \omega\left(r_{p j}\right) \alpha_{p}^{\mathrm{n}}}{\sum_{\mathrm{j}=1}^{\mathrm{N}} \omega\left(r_{p j}\right)}
$$

where $\omega\left(r_{p j}\right)$ is the linear weight factor with the form

$$
\omega\left(r_{p j}\right)=\left\{\begin{array}{ccc}
r_{\min }-r_{p j} & \text { for } & r_{p j}<r_{\min } \\
0 & \text { for } & r_{p j}>r_{\min }
\end{array},\right.
$$

where $r_{\min }$ denotes the radius of the filter, which identifies the nodal sensitivity numbers which will influence the sensitivity of the $p^{\text {th }}$ element. As shown in Fig. 2. nodes located inside the circular shadow area will be used in the filter scheme for the $i^{\text {th }}$ element (red one). 


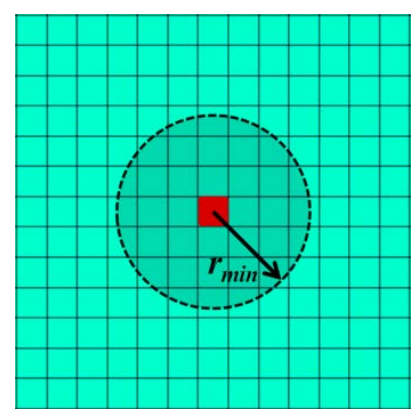

Fig. 2 Diagrammatic sketch of filter scheme

In order to ensure a stability of the iteration process, after the first iteration, the sensitivity number is further modified as[7, 47, 48]:

$$
\tilde{\alpha}_{p, h}=\frac{1}{2}\left(\alpha_{p, h}^{m}+\tilde{\alpha}_{p, h-1}\right)
$$

where $\alpha_{p, h}^{m}$ is the sensitivity number of the $p^{\text {th }}$ pixel in the $h^{\text {th }}$ iteration which is given by Eq. (12). The design variable $\mathrm{x}_{p}$ will be reset to 0 or 1 according to the their sensitivity number $\left(\tilde{\alpha}_{p, h}\right)$ rankings. When $\mathrm{V}_{h}$ is equal or larger to $\mathrm{V}_{f}^{*}$, BESO/FEM procedure will stop if the following convergence criterion is achieved:

$$
\text { error }=\frac{\left|\sum_{i=1}^{N} f_{h-i+1}-f_{h-N-i+1}\right|}{\sum_{i=1}^{N} f_{h-i+1}} \leq \tau
$$

where $\mathrm{N}$ is an integer number defined to be 3 which means that the fluctuation of the mean optimized results in the last 6 iterations is within acceptable limits. $f$ is the proposed objective function and $\tau=0.1$ is allowable convergence tolerance; $h$ is the iteration number. The flow chart for the BESO/FEM algorithm used in the PnCs topologic optimization is outlined as 


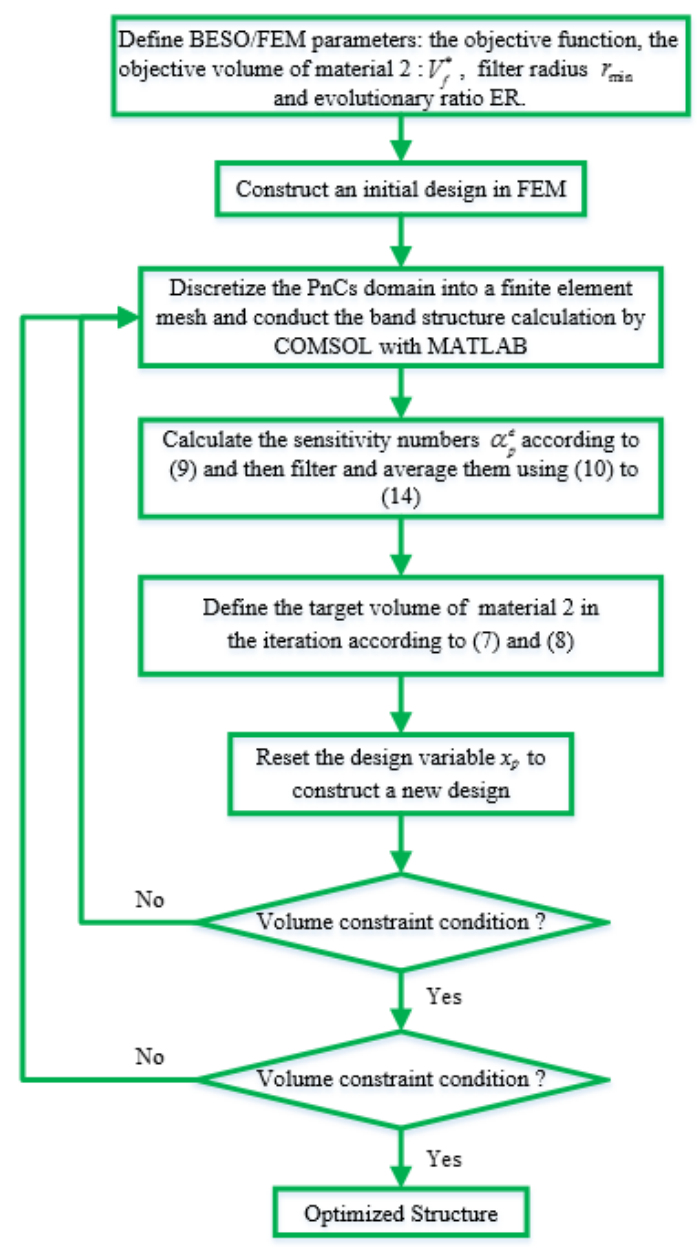

Fig. 3. Flow chart of BESO/FEM procedure for PnCs topological optimization

\section{Numerical results}

In order to show the robustness and computational efficiency of the BESO/FEM method, we conducted the bandgap optimizations of the 2D square-latticed solid PnCs with different material combinations (Au/Epoxy and $\mathrm{Pb} /$ Epoxy). In the optimizations program, the unit cell was divided into $20 \times 20$ 4-node quadrilateral elements. Due to the computational simplification and the square-symmetry of the unit cell, only a quarter of the unit cell was modeled. In order to illustrate clearly, different colors (green and red) were used to denote the Material 1 and 2 in unit cells, respectively. The BESO procedure parameters used in this paper are given as follows: the filter radius $\mathrm{r}_{\min }$ is $\sqrt{2} \mathbf{a} / 10$, where $\mathbf{a}$ is the lattice constant $(|\mathbf{a}|=0.02 \mathrm{~m})$. The evolution rate ER is $0.02 . \omega$ indicates the normalized frequency $\omega_{\text {norma }} \mathbf{a} / 2 \pi V_{\mathrm{t}}$, where $V_{\mathrm{t}}=1160 \mathrm{~m} / \mathrm{s}$ is the transverse wave velocity in the epoxy (matrix material). For the 
$\mathrm{Au} /$ Epoxy system, the physical properties are: $\rho_{1}=1.18 \mathrm{~g} / \mathrm{cm}^{3} ; \lambda_{1}=4.43 \mathrm{GPa}$; and $\mu_{1}=1.59 \mathrm{GPa}, \rho_{2}=19.5 \mathrm{~g} / \mathrm{cm}^{3}, \lambda_{2}=$ 160.3 GPa; $\mu_{2}=29.9 \mathrm{GPa}$. For the Pb/Epoxy system, the physical properties are $\rho_{1}=1.18 \mathrm{~g} / \mathrm{cm}^{3} ; \lambda_{1}=4.43 \mathrm{GPa} ; \mu_{1}=$ $1.59 \mathrm{GPa} ; \rho_{2}=11.6 \mathrm{~g} / \mathrm{cm}^{3} ; \lambda_{2}=42.03 \mathrm{GPa}$ and $\mu_{2}=14.9 \mathrm{GPa}$.

The optimizations for PnCs started from a cell only made from matrix material (Material 1, Epoxy), which is shown in Fig. 4a. The corresponding initial and filtered sensitivity numbers of first two bandgaps for in-plane and out-of-plane modes are shown in Fig. 4b and 4c. The negative values indicate no band gap appears at that moment.

As shown in Fig. 4, for both of the in-plane and the out-of-plan modes, filter scheme shrunk the distribution of initial sensitivity numbers (red and green curves) by about 40 percent to obtain filtered sensitivity numbers (black and blue curves with symbols). Meanwhile, the initial sensitivity numbers are more oscillatory than the filtered ones, which could be seen from Fig. 4c. There are some small mutations appearing in the initial sensitivity curve (red curve), which could be filtered out as shown by the black curve with symbols. This ability of filter scheme significantly benefits the convergence of the proposed BESO/FEM program.
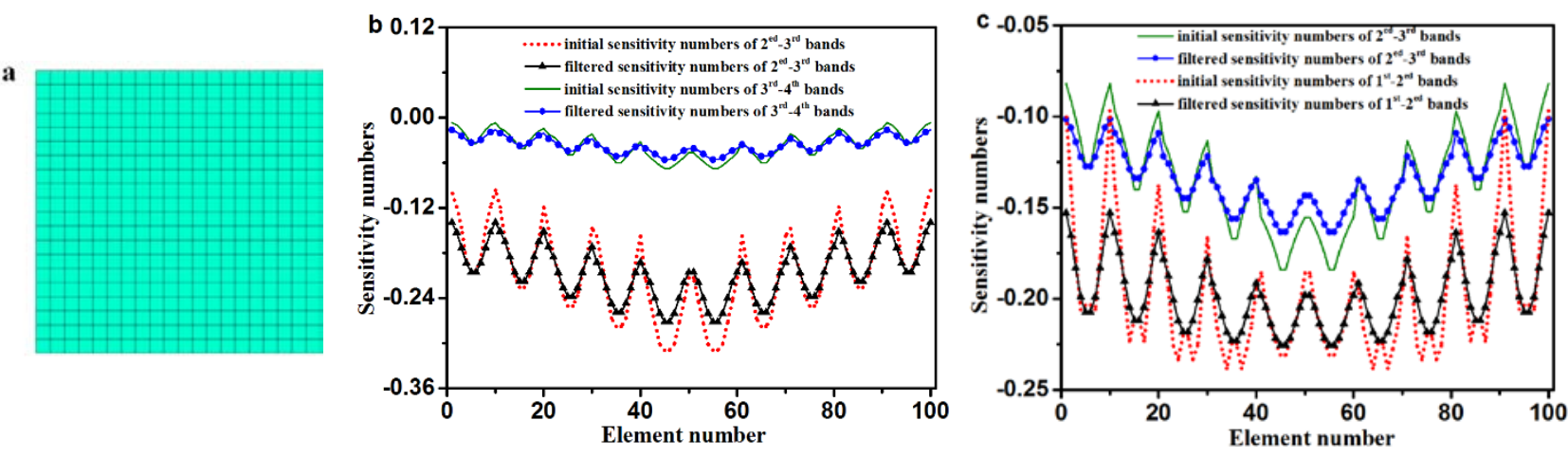

Fig. 4 (a) Initial design made of 4-node quadrilateral elements and the corresponding sensitivity numbers of each element for (b)

$\mathrm{Au} /$ Epoxy system in-plane mode and (c) Pb/Epoxy system out-of-plan mode.

\subsection{In-plane mode band gap optimization}

\subsubsection{Topological optimization of PnCs in different material volume fraction}


Firstly, with the aim to show the effect of the volume fraction on the optimization results, a comprehensive optimization of $\mathrm{Pb} / \mathrm{Epoxy}$ PnCs system is made between the volume fractions (Material 2) 0 to 1 . The objective of the optimization is the first bandgap (between $3^{\text {rd }}$ to $4^{\text {th }}$ bands). The initial design used in this optimization is the full design made of matrix material (Material 1, Epoxy) as shown in Fig. 4a.

The upper and lower edges of the $3^{\text {rd }}$ to $4^{\text {th }}$ bands are plotted in Fig. 5 by black dash line and black solid line. In the same volume fraction, if the upper edge is higher than the lower edge, there will be an ABG formed which is marked out by shadow parts. The optimized dimensionless gap size represented by the relative ratio of the bandgap width and the central frequency is shown by blue solid line. As shown in Fig.5, the ABG begins to appear at the volume fraction 0.06 . As the increase of the volume fraction, the ABG becomes wider and reaches the maximum value 0.474 at the volume fraction 0.38 , which is more than $1000 \%$ larger than it when volume fraction is 0.06 . Meanwhile, the dimensionless gap size also achieves its maximum value 0.6447, after which, the ABG is closed at the volume fraction 0.85. The results in Fig. 5 indicate that there exists an optimized volume fraction, at which the ABG has the maximum value and largest dimensionless gap size.

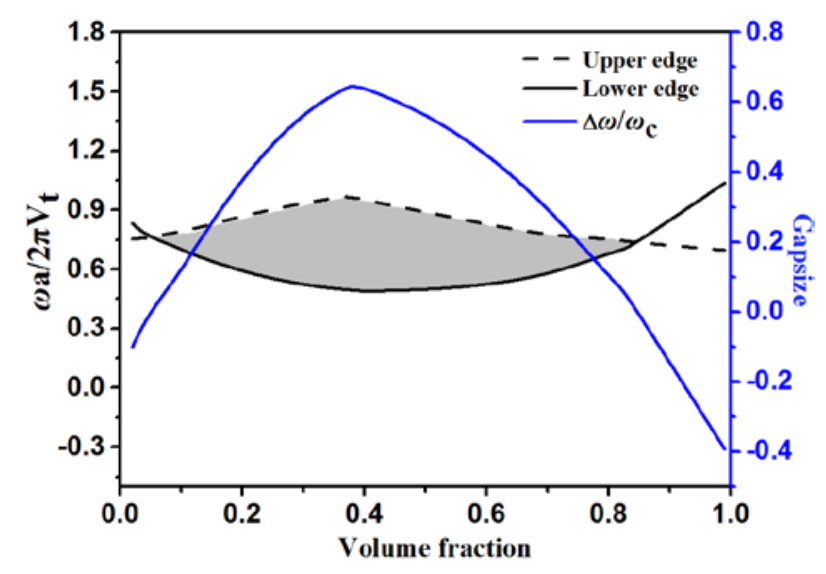

Fig. 5. The $3^{\text {rd }}$ to $4^{\text {th }}$ bandgaps of Optimized Pb/Epoxy PnCs in different volume fractions.

\subsubsection{Topological optimization of PnCs in different matrix/scatter materials.}

With the aim to examine the effect of different material combinations on the optimization, we conducted the 
optimization work of the first bandgap in $\mathrm{Pb} /$ Epoxy system and $\mathrm{Au} /$ Epoxy system. It is known that at the $\Gamma$ point (Fig. 1), the first two eigenmodes are rigid one and the minimum eigen-frequencies at the first and second bands are both zero. Consequently, there is no bandgap between the first and second bands. Here the ABG between the $2^{\text {nd }}$ $3^{\text {rd }}$ bands are optimized to maximize the band gap width. To verify the availability of our algorithm, the in-plane mode optimizations for the first absolute band gap (ABG) in both Pb/Epoxy and Au/Epoxy PnCs are made. The optimized results obtained by other optimization algorithm[41] are also given for comparison. The volume fraction of Material 2 was set as $V_{f}^{*}=0.11$. Optimized topologies of Au/Epoxy PnCs with its band structure are shown in Fig. 6.

In Fig. 6a, the resulted unit cell of Au/Epoxy PnCs and the corresponding $3 \times 3$ lattice are shown, in which red shadows represent Material $2(\mathrm{Au})$ and the green part is Material 1 (Epoxy). The optimized unit cell is one rectangle Au inclusion with four round corners and a slightly longer longitudinal axis embedded in Epoxy matrix. Figure 6 shows the corresponding band structures for the optimized Au/Epoxy PnCs. The shaded stripe represents the $2^{\text {nd }}-3^{\text {rd }}$ ABG. The gap ratio of the bandgap $\Delta \omega$ to its center frequency $\omega_{c}$ for the the $2^{\text {nd }}-3^{\text {rd }} A B G$ is up to 0.166 , it is higher than 0.151 obtained in the same volume fractions for the Pb/Epoxy system shown in Fig.5.

a
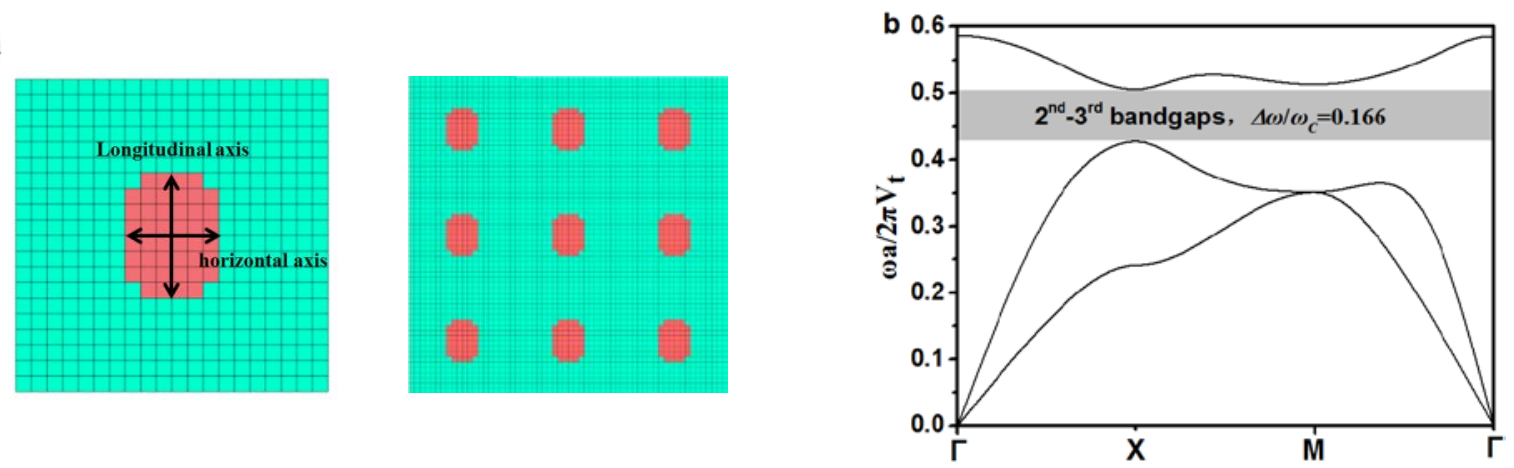

Fig. 6. Optimized Au/Epoxy PnCs structures (a) and the corresponding band structure (b) for the $2^{\text {nd }}-3^{\text {rd }} A B G$ with $V_{f}^{*}=0.11$.

Fig. 7 shows the evolution histories of the band size with corresponding cell topologies. The left vertical axis shows the dimensionless frequency meanwhile the right one is the filling fraction of Material 2. Negative values of 
$\Delta \omega$ mean that there is no bandgap at that point. In Fig. 7 blue line shows the filling fraction of Material 2 while black and red ones represent the gap width $(\Delta \omega)$ and the gap ratio $\Delta \omega / \omega_{c}$. It is seen that as the volume fraction gradually increasing to the predefined reference value $\mathrm{V}_{f}^{*}=0.11$, the gap width $\Delta \omega$ and the gap ratio progressively grow from negative values to positive ones which means that the ABGs is opened. The values are gradually increased with the increase of the filling fraction of Material 2, which corresponds that an Au inclusion starts from a single spot in the center of the lattice to an optimal topology. As shown in Fig. 7, volume constraint condition is satisfied at 0.11 at the $6^{\text {th }}$ iterations, after which, $\Delta \omega$ (black line) and $\Delta \omega / \omega_{c}$ (red line) tend to constant values which means that volume fraction is no longer changed and the convergence criterion is achieved to obtain the optimized topology. The results are also shown that BESO/FEM procedure is robust enough to converge to the expected value with the $20 \times 20$ 4-node mesh.

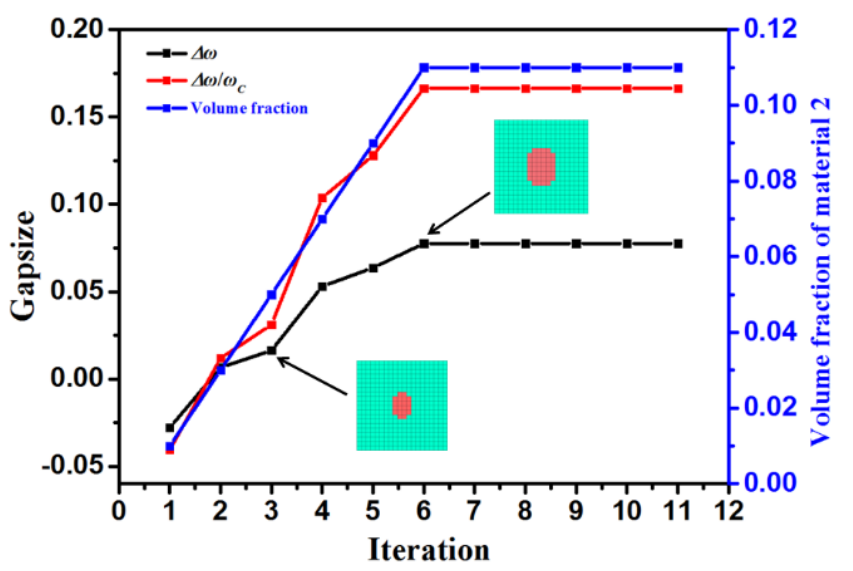

Fig. 7. Evolution histories of the $2^{\text {nd }}-3^{\text {rd }}$ bandgap and the volume fraction of material 2 for Au/Epoxy PnCs

For the purpose of validating the optimized results, the transmission spectra of the optimized Au/Epoxy PnCs structures along $\Gamma X$ direction are displayed in Fig. 8. It can be seen that the transmission spectra of the obtained structures coincide well with the band structures, which is further indicates the accuracy of the calculation. 

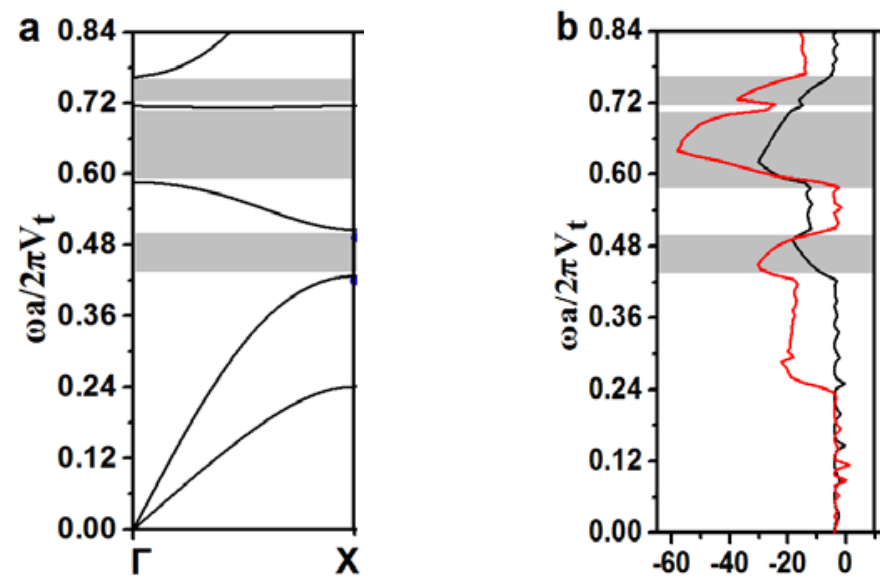

Fig. 8. The band structure (a) and transmission spectra (b) of the optimized Au/Epoxy PnCs structure along $\Gamma X$ direction.

In the previous section, we know that the max first dimensionless gapsize of $\mathrm{Pb} / \mathrm{Epoxy} \mathrm{PnCs}$ is achieved in the volume fraction 0.38, the corresponding the optimized topologies of PnCs and the corresponding band structure are displayed in Fig. 9.

As shown in Fig. 9a, in the optimized unit cell, there are two trapezoidal Pb inclusion symmetrical embedded in Epoxy on opposite sides of the unit cell. Fig. 9b shows the corresponding band structures, with the shaded broad stripe representing the $3^{\text {rd }}-4^{\text {th }}$ bandgap. It is seen that the ABG bandwidth of the optimized structure is 0.6447 . It is $41.69 \%$ bigger than the result, 0.455 , obtained by a genetic topological optimization algorithm[41] with the filling fraction 0.35 .

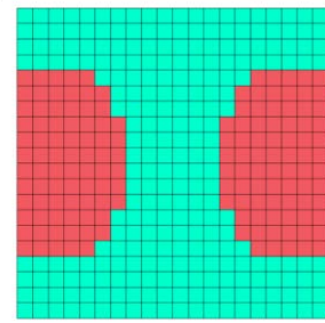

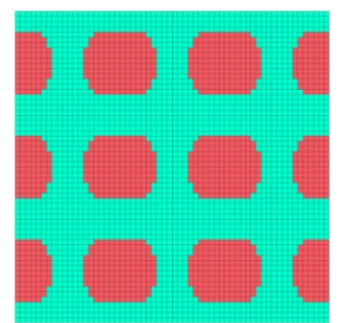

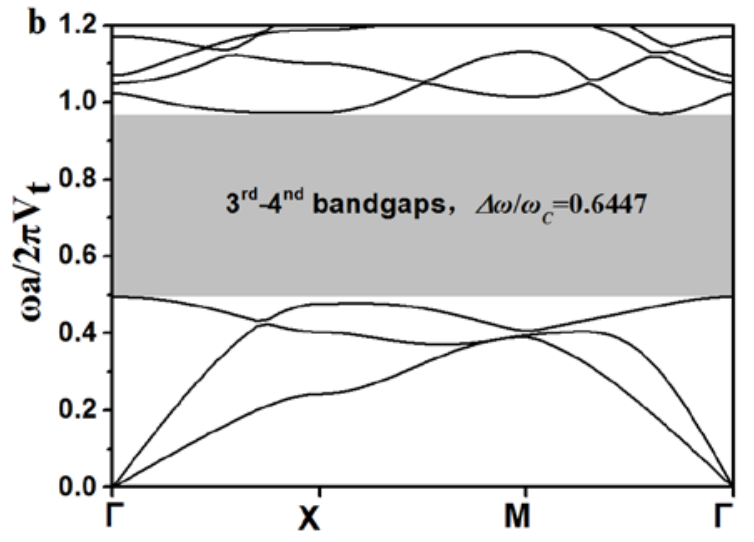


Fig. 9. Optimized Pb/Epoxy PnCs structures (a) and the corresponding band structures (b) for the $3^{\text {rd }}-4^{\text {th }}$ bandgaps with the constraints

$$
\text { of } \mathrm{V}_{f}^{*}=0.38
$$

Fig. 10. shows the evolution histories of the proposed BESO/FEM optimization for maximizing the width of the $3^{\text {rd }}-4^{\text {th }}$ ABG. As shown in Fig. 10, before the $16^{\text {th }}$ iteration, both the bandgap width $(\Delta \omega)$ and the dimensionless gap ratio $\left(\Delta \omega / \omega_{\mathrm{c}}\right)$ increase along with the increase of the filling fraction of Material 2. It is noted that at the $16^{\text {th }}$ to the $18^{\text {th }}$ iterations, there is a drop in the dimensionless gap ratio. This is because of a relative increase of the center frequency at the $16^{\text {th }}$ to the $18^{\text {th }}$ iterations which leads to the drop of the dimensionless bandwidth. Volume constraint condition is satisfied at 0.38 at $19^{\text {th }}$ iterations, after which, the bandgap width $(\Delta \omega)$ and corresponding dimensionless gap ratio $\left(\Delta \omega / \omega_{\mathrm{c}}\right)$ tend to be stable with minor fluctuations at the next ten iterations until convergence criterion is achieved and then the optimization program is stopped.

Due to the complexity of the optimization work in wave propagation problems, local optimal solutions is easy to occur in the iterative process; those fake 'optimal' structures may lead the whole optimization process to a path away from the optimal results in evolutionary algorithm. As shown in Fig. 10, at early iterations, there is a scatter in the center of the unit cell which is marked out by black dotted lines. Compared to the final optimized structure, it is obvious that this scatter is a temporary fake 'optimal' structure; BESO/FEM procedure deletes it at the $8^{\text {th }}$ iteration and converges at the $20^{\text {th }}$ iteration. By the remove of this scatter in the center, BESO/FEM can generate a more optimized structure with a larger bandgap in a more accurate way. Compared with other optimization method such as Evolutionary Structural Optimization (ESO)[28], deleting existing structures in BESO method is a key ability to solve such problem in the optimization procedure to guarantee the stability of the convergence of the optimization process to optimal direction. For the topological optimization of Pb/Epoxy PnCs, GA algorithm also got the similar results[41]. But compared to approximately a thousand iterations in the same task needed by the genetic topological optimization algorithm, the proposed BESO optimization displays its higher computational efficiency. 


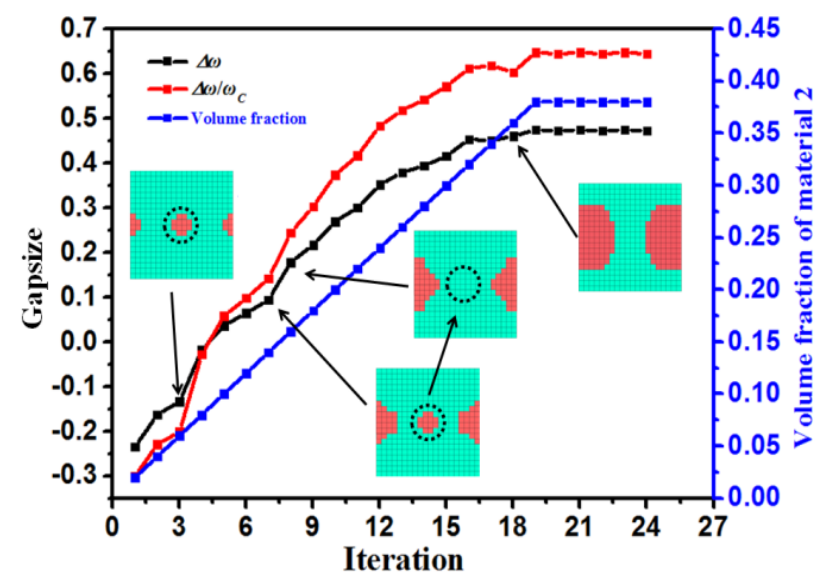

Fig. 10. Evolution histories of the $3^{\text {rd }} 4^{\text {th }}$ bandgap and the volume fraction of material 2 for Pb/Epoxy PnCs

\subsubsection{Topological optimization of PnCs from different initial designs.}

To further show the robustness and effectiveness of the proposed BESO/FEM procedure, it is informative to consider the influence of different initial designs on the optimized results.

Here, three initial designs of Au/Epoxy PnCs, which are shown in Fig. 11, are used to start the cell optimization. Initial design a (Fig. 11a) was the one used in the previous calculation started from a pure cell made from material 1. Initial design b (Fig. 11b) was an initial design based on the resulting topology of Au/Epoxy PnCs for the $2^{\text {nd }}-3^{\text {rd }}$ ABG. Initial design c (Fig. 11c) is based on the initial design b with the central Au scatter divided into two parts. To start the optimization procedure, the volume fraction $\mathrm{V}_{f}^{*}=0.39$ was applied to Material 2.
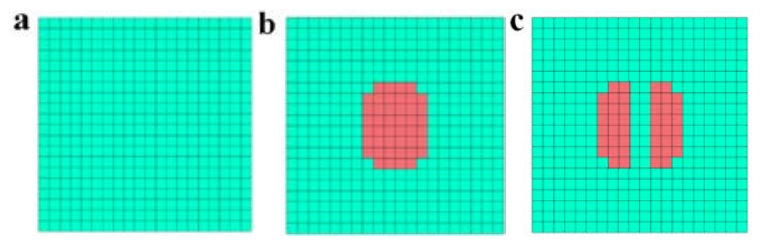

Fig. 11. Initial designs for maximizing the $3^{\text {rd }}-4^{\text {th }}$ bandgap of Au/Epoxy PnCs

Fig. 12 shows the finial optimized topologies for the $3^{\text {rd }}-4^{\text {th }}$ ABGs of Au/Epoxy PnCs from the initial designs a, $\mathbf{b}$ and $\mathbf{c}$, respectively. It is seen that different initial designs lead to the same optimized results, that is, a rectangular Au scatter embedded in Epoxy matrix. The corresponding band structures for the optimized Au/Epoxy PnCs are 
given in Fig. 12 b, $d$ and f. It is clear that the band structures of the three optimized unit cells from the different initial designs are almost the same as expected. The bandgap ratio are up to 0.912 , which is very close to 0.902 given by genetic topological optimization algorithm[41].

a

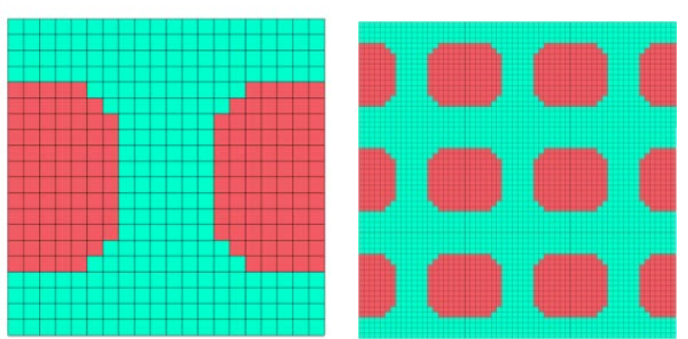

c
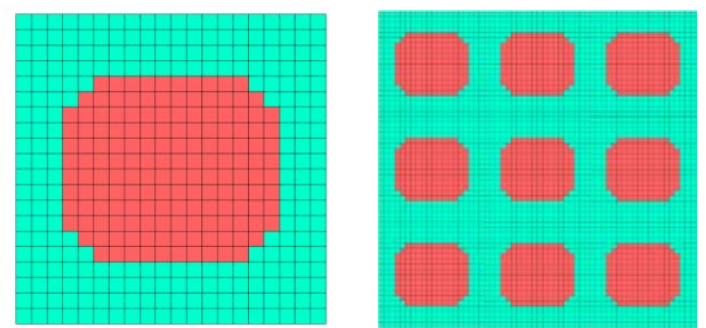

$\mathbf{e}$

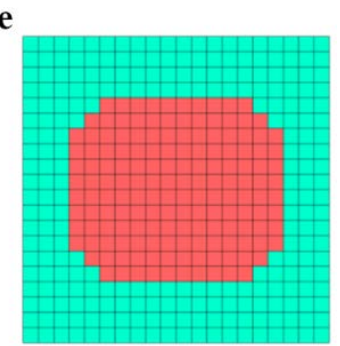

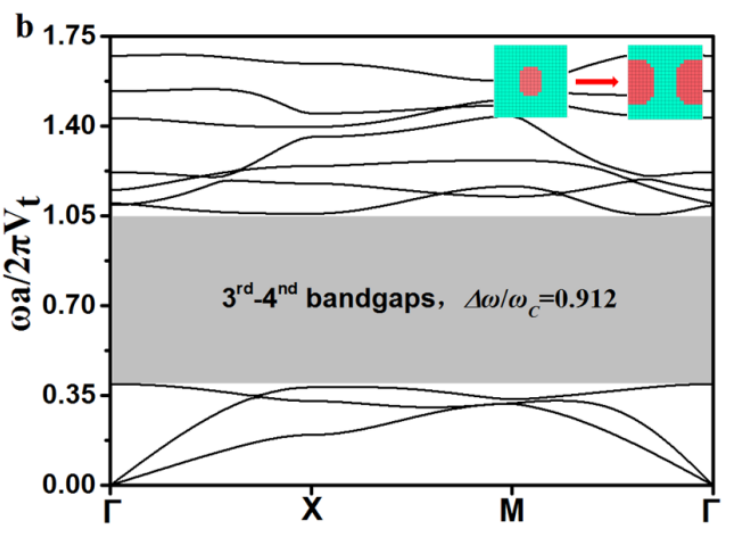
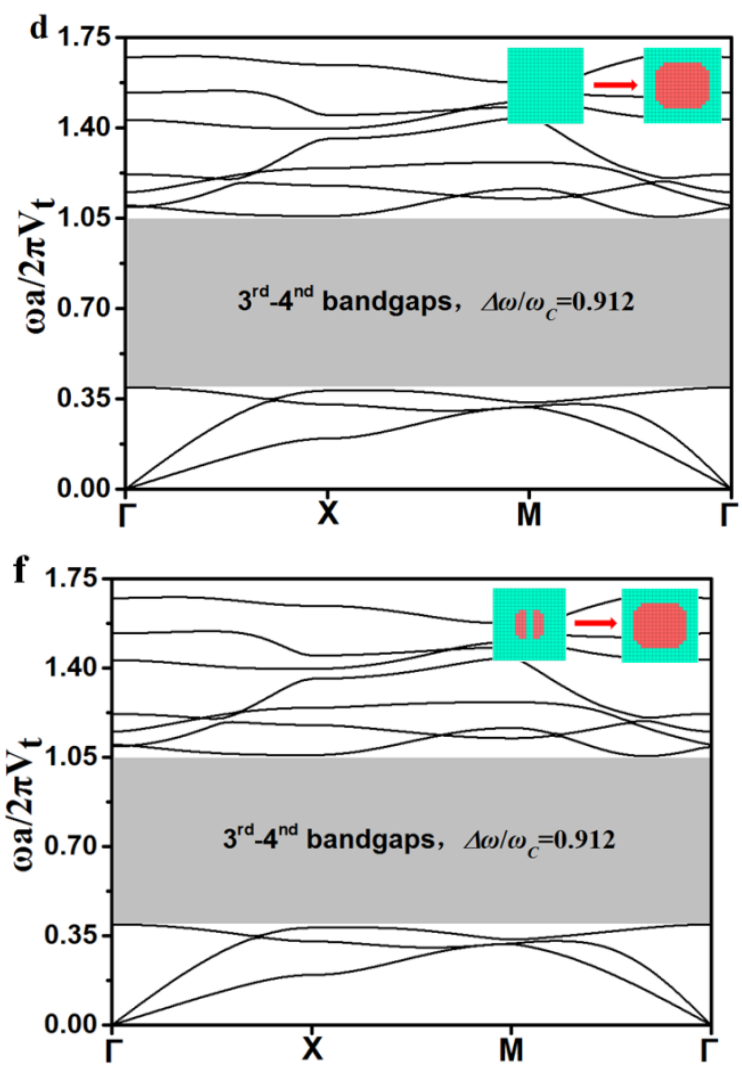

Fig. 12. Optimized Au/Epoxy PnCs structures and the corresponding band structures for the $3^{\text {rd }}-4^{\text {th }}$ bandgaps from different initial designs with the constraints of $\mathrm{V}_{f}^{*}=0.39$.

Figure 13 shows evolution histories of the proposed BESO optimization for maximizing the bandgap from the initial designs a (Fig. 11a), b (Fig. 11b) and c (Fig. 11c) respectively. As shown in Fig. 13a, the width of bandgap 
(black curve) from the initial design a is increased along with the increase of the filling fraction of Material 2. It should be noted that a temporary attenuation of the dimensionless bandgap width occurred at the $3^{\text {rd }}-4^{\text {th }}$ iterations, the proposed BESO procedure deleted some elements and separated the scatter to two parts and moved them to the side of the unit cells. After that, volume constraint condition is achieved at the $20^{\text {th }}$ iterations, the convergence criterion is satisfied the after 5 more iterations.

For the optimization procedure from the initial design $\mathbf{b}$, scatters in the center of the unit cell gradually grow lager, volume fraction evolves to the constraint condition 0.39 at the $15^{\text {th }}$ iterations and the procedure convergence criterion is achieved at the $20^{\text {th }}$ iterations. As the optimization procedure from initial design c (Fig. $11 \mathrm{c}$ ), both of the bandgap width $\Delta \omega$ (black line) and the corresponding dimensionless value $\Delta \omega / \omega_{\mathrm{c}}$ (red line) increase with the increasing of the filling fraction of Material 2. Scatters in the center of the unit cell gradually evolve to the optimized structures within only 20 iterations. It is seen that although started with different initial designs, the proposed BESO/FEM procedure can efficiently converge to the desired value within 25 iterations.

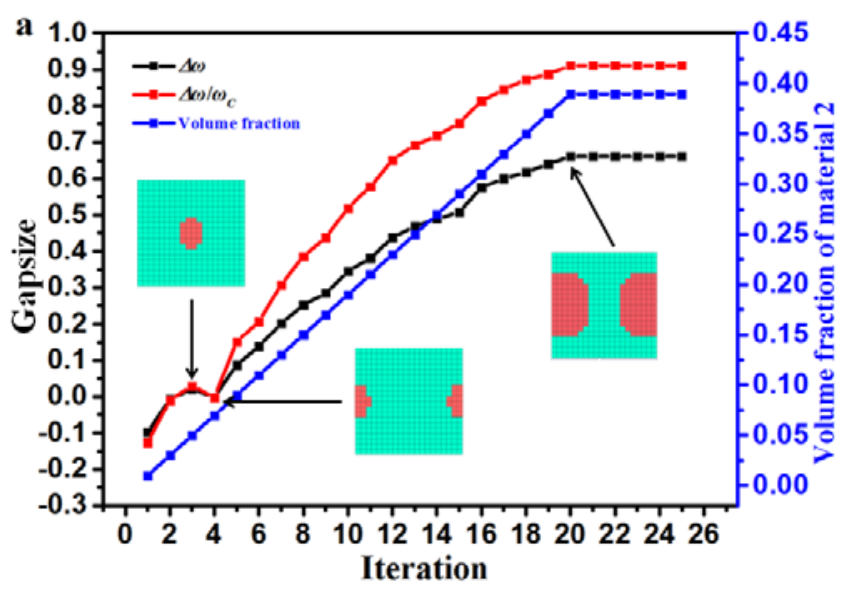



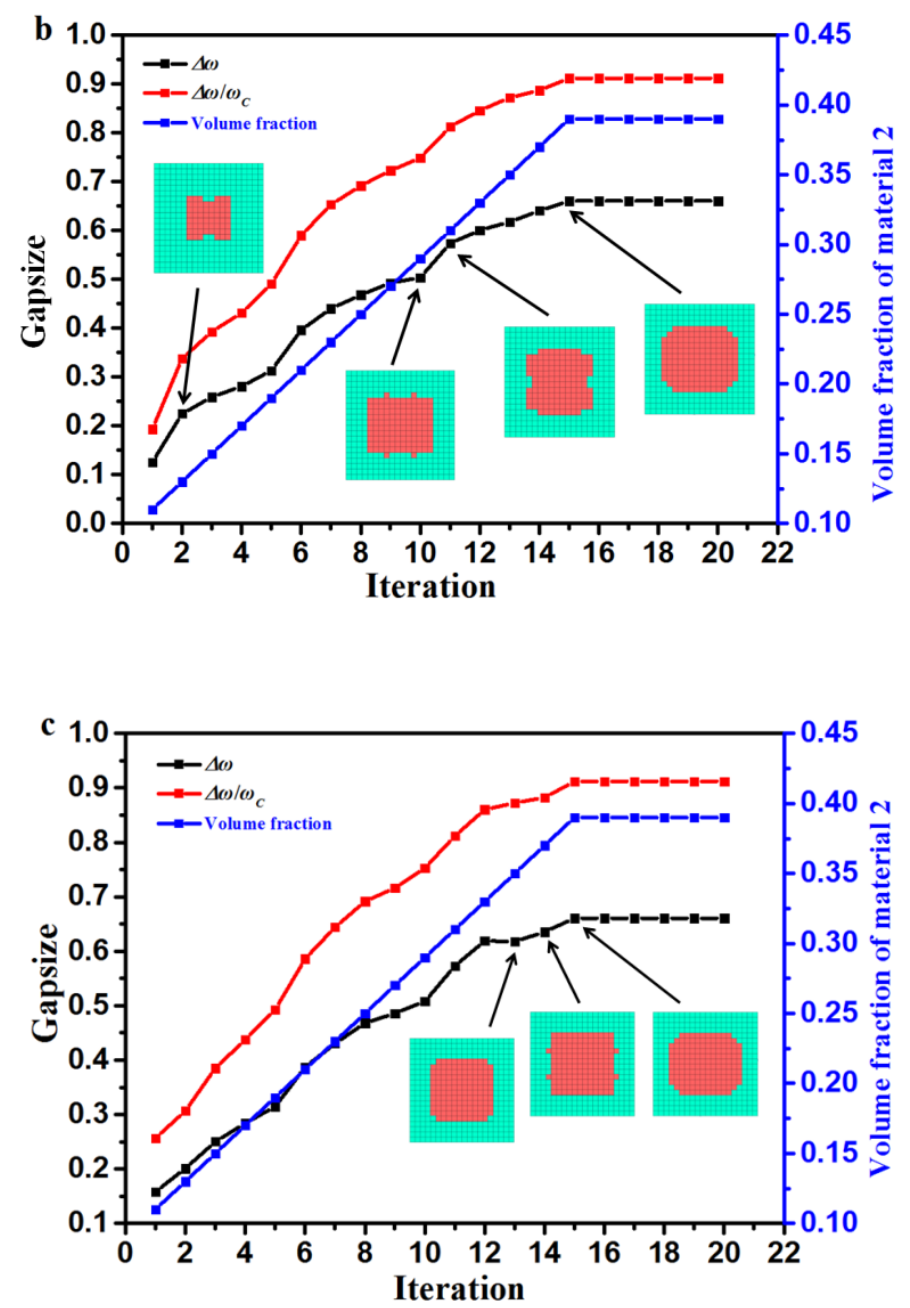

Fig. 13. Evolution histories of the $3^{\text {rd }}-4^{\text {th }}$ bandgap and the volume fraction of material 2 for Au/Epoxy PnCs; (a), (b) and (c) are the results started from initial design a, b and c, respectably.

\subsection{Out-of-plane mode band gap optimization}

In this section, the out-of-plane mode bandgap optimization in $\mathrm{Pb} /$ Epoxy system is made. In the calculation, the volume constraint is set as $\mathrm{V}_{f}^{*}=0.32$, initial design is a full design made of material 1 shown as Fig4 $\mathrm{a}$. The optimized structures for the first to the fourth ABGs and their corresponding band structures are shown in Fig. 14. The corresponding $3 \times 3$ lattice is given with the central unit cells marked out by the black dash lines.

The results show that along with the increase of the ABG band order, the optimized topologies also become complicated. As illustrated in Fig. 14a, the optimized unit cell corresponding to the first ABG is a rectangular Pb scatter in matrix. When the objective band turns to the second ABG as shown in Fig. 14b, there are one lager square 
scatter in the central of the unit cell with other four $1 / 4$ square scatters at the four unit cell corners. For the optimized third ABG shown in Fig. 14c, there are two smaller approximately elliptical scatters in the central of the unit cell with two half larger ones on the two horizontal opposite sides. Fig. $14 \mathrm{~d}$ shows the optimized $3 \times 3$ lattice structures corresponding to the fourth ABG and its band structures. Two approximately circular scatters are symmetrically distributed in the center with four half scatters distributed on the upper and lower boundaries. It is found that the scatter number in one unit cell equals to the ABG band order. The predicted structures for the optimal PnC structures in Pb/Epoxy system based on BESO algorithm are similar to those given by Sigmund[49] and Dong[41].
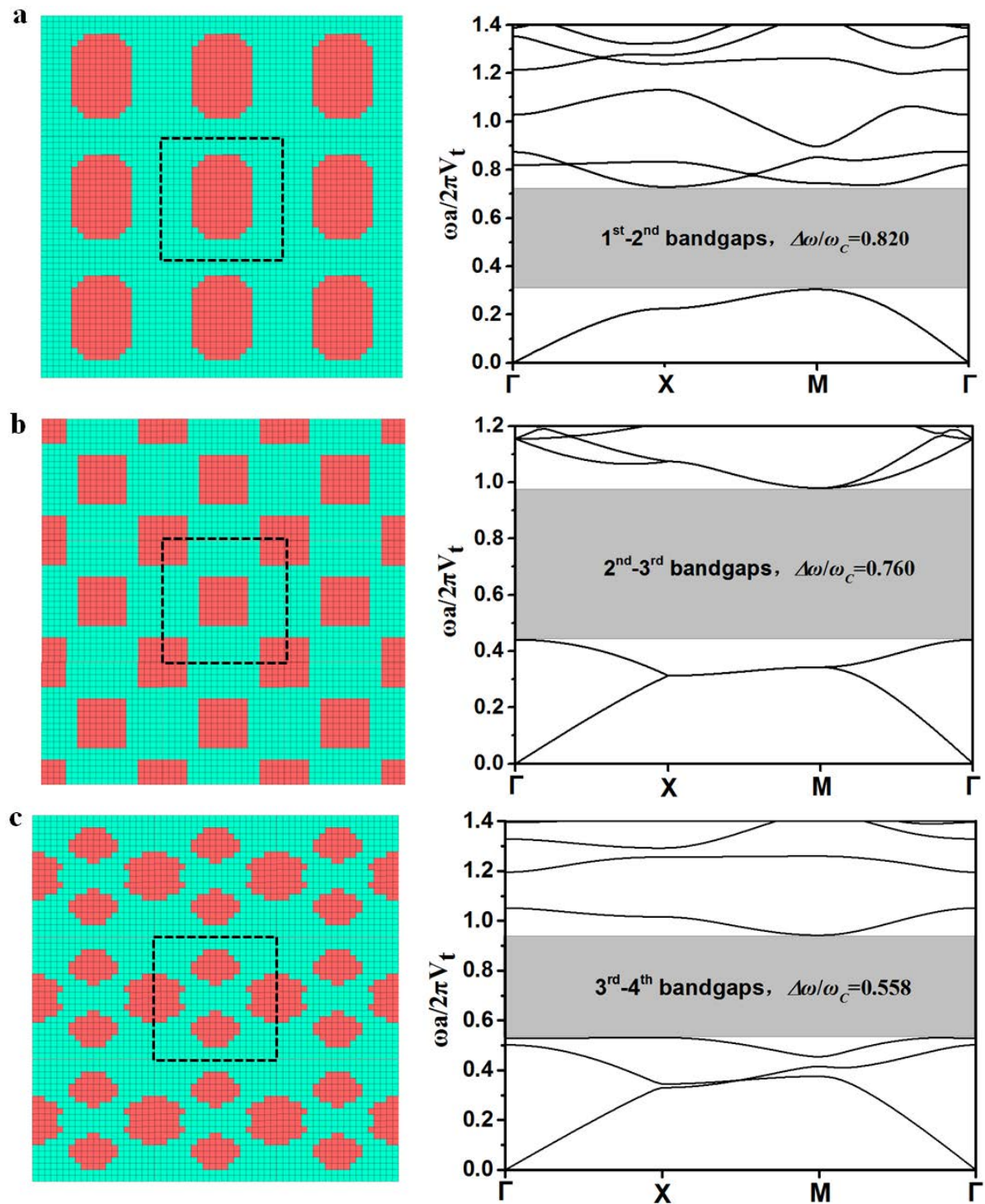

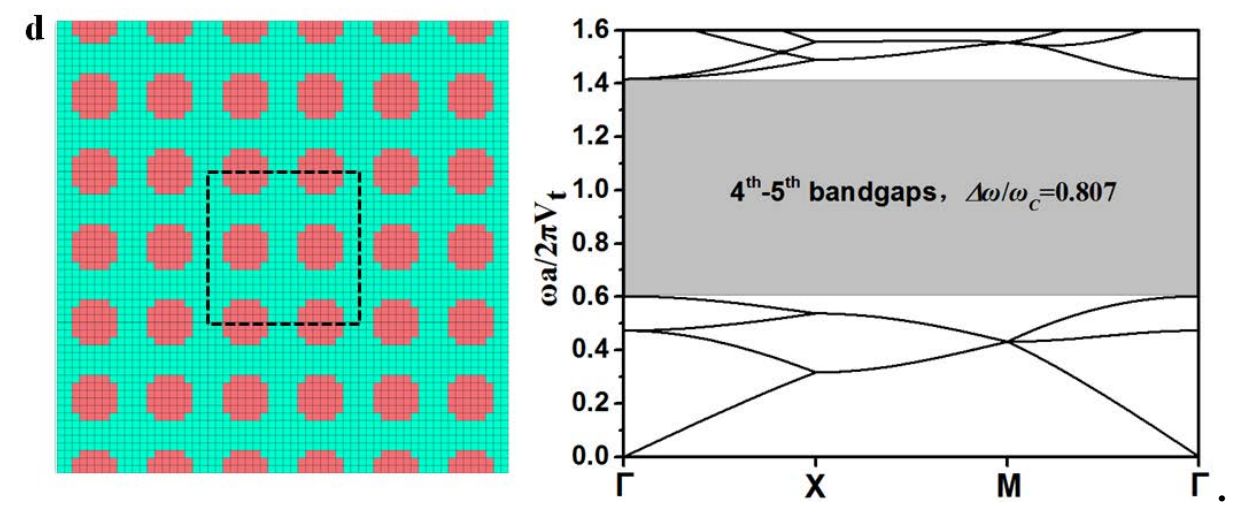

Fig. 14. Optimized $3 \times 3$ lattice structures of Pb/Epoxy system and the corresponding band structures for the first to fourth bandgaps

in out-of-plane motion.

\section{Conclusions}

In this paper, a combined BESO/FEM optimization algorithm is proposed for maximizing the ABG bandwidth between a given pair of adjacent bands for both the in-plane wave and out-of-plane modes. Summarizing the results above, we can conclude that:

1. In the proposed BESO/FEM procedure, no specific guess (seeding) designs are needed. By adding and removing material simultaneously in the evolutionary process, BESO/FEM gradually evolved the topology of unit cells to optimal ones in a high robustness and computational efficiency way. Ultrawide ABG is obtained in both in-plane and out-of-plane wave modes.

2. Filter scheme proposed based on the statics is integrated into the proposed BESO/FEM procedure, results proved that it is also highly effective in solving the elastic wave optimization problems.

3. Based on the proposed BESO/FEM procedure, topological optimization from different initial designs will result in the same maximum bandgap.

4. Volume fraction of material have a significant influence on the optimized bandgaps, for the case that maximizing the bandgap without considering a specific volume constraint, there exists an optimized volume 
fraction, at which ultrawide ABG can been obtained.

5. Compared to the results obtained by other optimization approaches under the same or similar volume fraction, the proposed BESO/FEM optimization algorithm can result in larger dimensionless bandgaps which will be useful for the design of acoustic materials.

\section{Acknowledgements}

The financial supports from the National Natural Science Foundation of China (Grant No. 11772044) and the National Basic Research Program of China (973 Program) (Grant No. 2015CB057800) are acknowledged.

\section{References}

[1] M.S. Kushwaha, P. Halevi, L. Dobrzynski, B. Djafari-Rouhani, Acoustic band structure of periodic elastic composites, Physical review letters, 71 (1993) 2022-2025.

[2] M. Kushwaha, B. Djafari-Rouhani, Sonic stop-bands for periodic arrays of metallic rods: honeycomb structure, Journal of sound and vibration, 218 (1998) 697-709.

[3] S. Yang, Y. Liu, T.S. Liang, Band structures in the nematic elastomers phononic crystals, Physica B, 506 (2017) 55-

64.

[4] J.K. Huang, Z.F. Shi, W.X. Huang, Multiple band gaps of phononic crystals with quasi-Sierpinski carpet unit cells, Physica B, 516 (2017) 48-54.

[5] M.B. Nouri, M. Moradi, Presentation and investigation of a new two dimensional heterostructure phononic crystal to obtain extended band gap, Physica B, 489 (2016) 28-32.

[6] Z.Z. Yan, C.Q. Wei, H. Zheng, C.Z. Zhang, Phononic band structures and stability analysis using radial basis function method with consideration of different interface models, Physica B, 489 (2016) 1-11.

[7] K. Wang, Y. Liu, B. Wang, Follow-up optimized phononic band structure design based on reserved inclusions, 
Physica B: Condensed Matter, (2019).

[8] J.-F. Barthelemy, R.T. Haftka, Approximation concepts for optimum structural design—a review, Structural optimization, 5 (1993) 129-144.

[9] R.T. Haftka, H.M. Adelman, An analytical investigation of shape control of large space structures by applied temperatures, AIAA journal, 23 (1985) 450-457.

[10] N. Zhu, Y.L. Wang, Y. Zhu, L. Yang, N. Yu, Y.L. Wei, H.P. Zhang, A.D. Sun, Design of a treatment chamber for low-voltage pulsed electric field sterilization, Innov Food Sci Emerg, 42 (2017) 180-189.

[11] P. Jiang, X.P. Wang, T.N. Chen, J. Zhu, Band gap and defect state engineering in a multi-stub phononic crystal plate, J Appl Phys, 117 (2015).

[12] J. Zhu, Y.Q. Liu, Z.X. Liang, T.N. Chen, J.S. Li, Elastic Waves in Curved Space: Mimicking a Wormhole, Physical review letters, 121 (2018).

[13] X.P. Wang, P. Jiang, T.N. Chen, J. Zhu, Tuning characteristic of band gap and waveguide in a multi-stub locally resonant phononic crystal plate, Aip Adv, 5 (2015).

[14] Y. Liu, J.Y. Su, L.T. Gao, The influence of the micro-topology on the phononic band gaps in 2D porous phononic crystals, Phys Lett A, 372 (2008) 6784-6789.

[15] Y. Liu, J.Y. Su, Y.L. Xu, X.C. Zhang, The influence of pore shapes on the band structures in phononic crystals with periodic distributed void pores, Ultrasonics, 49 (2009) 276-280.

[16] L. Yang, N. Yang, B. Li, Extreme low thermal conductivity in nanoscale 3D Si phononic crystal with spherical pores, Nano letters, 14 (2014) 1734-1738.

[17] K. Wang, Y. Liu, T.S. Liang, Band structures in Sierpinski triangle fractal porous phononic crystals, Physica B, 498 (2016) 33-42.

[18] K. Wang, Y. Liu, Q.S. Yang, Tuning of band structures in porous phononic crystals by grading design of cells, 
Ultrasonics, 61 (2015) 25-32.

[19] K. Wang, Y. Liu, T. Liang, B. Wang, Band structures in fractal grading porous phononic crystals, Journal of Physics and Chemistry of Solids, 116 (2018) 367-374.

[20] Y.F. Chen, F. Meng, G.Y. Sun, G.Y. Li, X.D. Huang, Topological design of phononic crystals for unidirectional acoustic transmission, Journal of Sound and Vibration, 410 (2017) 103-123.

[21] A.L. Chen, L.Z. Tian, Y.S. Wang, Band structure properties of elastic waves propagating in the nanoscaled nearly periodic layered phononic crystals, Acta Mech Solida Sin, 30 (2017) 113-122.

[22] S.G. Zuo, H.D. Huang, X.D. Wu, M.H. Zhang, T.X. Ni, Low-frequency band gap of locally resonant phononic crystals with a dual-base plate, J Acoust Soc Am, 143 (2018) 1326-1332.

[23] M.P. Bendsøe, N. Kikuchi, Generating optimal topologies in structural design using a homogenization method, Computer methods in applied mechanics and engineering, 71 (1988) 197-224.

[24] G. Allaire, F. Jouve, A.M. Toader, Structural optimization using sensitivity analysis and a level-set method, J Comput Phys, 194 (2004) 363-393.

[25] M.Y. Wang, X. Wang, D. Guo, A level set method for structural topology optimization, Computer methods in applied mechanics and engineering, 192 (2003) 227-246.

[26] M.P. Bendsøe, Optimal shape design as a material distribution problem, Structural optimization, 1 (1989) $193-202$.

[27] M. Zhou, G. Rozvany, The COC algorithm, Part II: topological, geometrical and generalized shape optimization, Computer Methods in Applied Mechanics and Engineering, 89 (1991) 309-336.

[28] Y.M. Xie, G.P. Steven, A simple evolutionary procedure for structural optimization, Computers \& structures, 49 (1993) 885-896.

[29] X. Huang, Y.M. Xie, B. Jia, Q. Li, S.W. Zhou, Evolutionary topology optimization of periodic composites for extremal magnetic permeability and electrical permittivity, Structural and Multidisciplinary Optimization, 46 (2012) 
385-398.

[30] X.Y. Yang, Y.M. Xie, G.P. Steven, O.M. Querin, Bi-directional evolutionary structural optimization, in:

Proceedings of the 7th AIAA/USAF/NASA/ISSMO Symposium Multidisc Anal. Optim (St. Louis), 1998, pp. 1449-

1457.

[31] X. Guo, W.S. Zhang, J. Zhang, J. Yuan, Explicit structural topology optimization based on moving morphable components (MMC) with curved skeletons, Computer Methods in Applied Mechanics and Engineering, 310 (2016) 711748.

[32] W.S. Zhang, J. Yuan, J. Zhang, X. Guo, A new topology optimization approach based on Moving Morphable Components (MMC) and the ersatz material model, Structural and Multidisciplinary Optimization, 53 (2016) 12431260.

[33] J.K. Guest, A. Asadpoure, S.-H. Ha, Eliminating beta-continuation from heaviside projection and density filter algorithms, Structural and Multidisciplinary Optimization, 44 (2011) 443-453.

[34] O. Sigmund, A 99 line topology optimization code written in Matlab, Structural and multidisciplinary optimization, 21 (2001) 120-127.

[35] O. Sigmund, J.S. Jensen, Systematic design of phononic band-gap materials and structures by topology optimization, Philos T Roy Soc A, 361 (2003) 1001-1019.

[36] A. Diaz, A. Haddow, L. Ma, Design of band-gap grid structures, Structural and Multidisciplinary Optimization, 29 (2005) 418-431.

[37] S. Halkjær, O. Sigmund, J.S. Jensen, Maximizing band gaps in plate structures, Structural and Multidisciplinary Optimization, 32 (2006) 263-275.

[38] J.H. Holland, Adaptation in natural and artificial systems : an introductory analysis with applications to biology, control, and artificial intelligence, University of Michigan Press, Ann Arbor, 1975. 
[39] O.R. Bilal, M.I. Hussein, Ultrawide phononic band gap for combined in-plane and out-of-plane waves, Phys Rev E, 84 (2011).

[40] M.I. Hussein, G.M. Hulbert, R.A. Scott, Dispersive elastodynamics of 1D banded materials and structures: analysis, Journal of Sound and Vibration, 289 (2006) 779-806.

[41] H.W. Dong, X.X. Su, Y.S. Wang, C.Z. Zhang, Topological optimization of two-dimensional phononic crystals based on the finite element method and genetic algorithm, Structural and Multidisciplinary Optimization, 50 (2014) 593-604.

[42] Y. fan Li, X. Huang, F. Meng, S. Zhou, Evolutionary topological design for phononic band gap crystals, Structural and Multidisciplinary Optimization, 54 (2016) 595-617.

[43] X. Huang, Z.H. Zuo, Y.M. Xie, Evolutionary topological optimization of vibrating continuum structures for natural frequencies, Computers \& Structures, 88 (2010) 357-364.

[44] C. Kittel, P. McEuen, P. McEuen, Introduction to solid state physics, Wiley New York, 1996.

[45] Q. Li, G.P. Steven, Y.M. Xie, A simple checkerboard suppression algorithm for evolutionary structural optimization, Structural and Multidisciplinary Optimization, 22 (2001) 230-239.

[46] O. Sigmund, J. Petersson, Numerical instabilities in topology optimization: A survey on procedures dealing with checkerboards, mesh-dependencies and local minima, Structural Optimization, 16 (1998) 68-75.

[47] X. Huang, Y.M. Xie, Convergent and mesh-independent solutions for the bi-directional evolutionary structural optimization method, Finite Elem Anal Des, 43 (2007) 1039-1049.

[48] X. Huang, M. Xie, Evolutionary topology optimization of continuum structures: methods and applications, John Wiley \& Sons, 2010.

[49] O. Sigmund, K. Hougaard, Geometric properties of optimal photonic crystals, Physical review letters, 100 (2008). 